\title{
Author Correction: Impaired perceptual learning in a mouse model of Fragile $X$ syndrome is mediated by parvalbumin neuron dysfunction and is reversible
}

Anubhuti Goel, Daniel A. Cantu, Janna Guilfoyle, Gunvant R. Chaudhari, Aditi Newadkar, Barbara Todisco, Diego de Alba, Nazim Kourdougli, Lauren M. Schmitt, Ernest Pedapati, Craig A. Erickson and Carlos Portera-Cailliau (D)

Correction to: Nature Neuroscience https://doi.org/10.1038/s41593-018-0231-0, published online 24 September 2018.

In the version of this article initially published, a sentence was omitted from the Acknowledgments.

Original:

The authors thank K. Cohan, S. Cohen, and M. Hong for help with early behavioral experiments; P. Golshani and M. Einstein for advice on mouse behavior; the Janelia GENIE project (for providing GCaMP6s); P. Yu for building custom lick ports; and D. Buonomano, A. Contractor, and J. Sweeney for feedback on the manuscript. K. Battista created the illustration in Fig. 1b. This work was supported by the following grants: W81XWH-17-1-0231 (USAMRMC, DOD), Developmental Disabilities Translational Research Program \#20160969 (John Merck), SFARI Award 295438 (Simons Foundation), and 5R01HD054453 (NICHD/NIH) awarded to C.P.-C.; K23 MH112936 (NIMH/NIH) to E.P.; a grant from the Fragile X Alliance of Ohio to C.A.E.; and U01 DD001185 (NCBDDD/NIH), U54 HD082008 (NICHD/NIH), and a grant from the Cincinnati Children's Hospital Research Foundation to E.P. and C.A.E.

Corrected:

The authors thank K. Cohan, S. Cohen, and M. Hong for help with early behavioral experiments; P. Golshani and M. Einstein for advice on mouse behavior; the Janelia GENIE project (for providing GCaMP6s); P. Yu for building custom lick ports; and D. Buonomano, A. Contractor, and J. Sweeney for feedback on the manuscript. The authors also thank P. Pellionisz and A. Cheng for help assembling the two-photon microscope. K. Battista created the illustration in Fig. 1b. This work was supported by the following grants: W81XWH-17-1-0231 (USAMRMC, DOD), Developmental Disabilities Translational Research Program \#20160969 (John Merck), SFARI Award 295438 (Simons Foundation), and 5R01HD054453 (NICHD/NIH) awarded to C.P.-C.; K23 MH112936 (NIMH/NIH) to E.P.; a grant from the Fragile X Alliance of Ohio to C.A.E.; and U01 DD001185 (NCBDDD/NIH), U54 HD082008 (NICHD/NIH), and a grant from the Cincinnati Children's Hospital Research Foundation to E.P. and C.A.E.

The error has been corrected in the HTML and PDF versions of the article.

Published online: 15 November 2018

https://doi.org/10.1038/s41593-018-0273-3

\section{Publisher Correction: Attention improves memory by suppressing spiking-neuron activity in the human anterior temporal lobe}

John H. Wittig Jr., Anthony I. Jang (D), John B. Cocjin, Sara K. Inati and Kareem A. Zaghloul(D)

Correction to: Nature Neuroscience https://doi.org/10.1038/s41593-018-0148-7, published online 21 May 2018.

In the version of this article originally published, the reference citations in the Methods section were misnumbered. This has now been corrected in the HTML and PDF versions of the paper.

Published online: 20 August 2018

https://doi.org/10.1038/s41593-018-0224-z 